Int. J. Dev. Biol. 58: 929-934 (2014)

doi: $10.1387 / \mathrm{ijdb} .140155 \mathrm{rp}$

\title{
Evolutionary trend for metamery reduction and gonad shortening in Anurans revealed by comparison of gonad development
}

\author{
RAFAL P. PIPREK' ${ }^{1}$, ANNA PECIO ${ }^{1}$, MALGORZATA KLOC ${ }^{2,3}$, JACEK Z. KUBIAK ${ }^{4,5}$ and JACEK M. SZYMURA ${ }^{1}$ \\ ${ }^{1}$ Department of Comparative Anatomy, Institute of Zoology, Jagiellonian University, Krakow, Poland, \\ ${ }^{2}$ Department of Surgery, The Houston Methodist Hospital, Houston, USA, ${ }^{3}$ The Houston Methodist Research \\ Institute, Houston, USA, ${ }^{4}$ CNRS, UMR 6290, Institute of Genetics and Development of Rennes, Cell Cycle Group, \\ F-35043, France and ${ }^{5}$ Université Rennes 1, UEB, UMS Biosit, Faculty of Medicine, F-35043 Rennes, France
}

\begin{abstract}
The gonads develop as the metameric mesodermal structures at the ventral surface of the mesonephroi. To study the evolutionary trends for anuran gonads, we performed comparative analysis of three species from the basal paraphyletic group -Archaeobatrachia (Bombina bombina, Xenopus laevis, Pelobates fuscus) and five species from more derived monophyletic Neobatrachia group (Hyla arborea, Bufotes viridis, Rana dalmatina, Rana arvalis, Rana temporaria). Light and scanning electron microscopy analysis showed that in Archaeobtrachia the undifferentiated gonads and ovaries, but not the testes, have pronounced external metamery. In contrast, Neobatrachia lacked external gonadal metamery and the internal metamery was well pronounced in the ovaries, but only rudimentary in the testes. There was also a difference in the site of genital ridge formation between these two groups. In Archaeobatrachia, the genital ridges developed along almost the entire length of mesonephroi, while in Neobatrachia they were limited to the anterior parts of the mesonephroi. A transient form was observed in P. fuscus, where the gonads formed along the anterior half of the mesonephroi. There was also an evolutionary trend for gradual postero-anterior shortening of the gonadal anlagen. In summary, our study indicates that among anurans there is an evolutionary trend for the gradual loss of metamery and shortening of the gonad.
\end{abstract}

KEY WORDS: Anura, testis, ovary, mesonephros, metamery

\section{Introduction}

The vertebrate gonads: ovaries and testes develop from the common sexually indifferent anlagen - the genital ridges (Wylie et al., 1976). The genital ridges arise as the parallel folds located at the ventral surface of mesonephroi along the dorsal mesentery. They are composed of somatic and primordial germ cells (PGCs) that in amphibians immigrate into the genital ridges from the gut (Wylie and Heasman, 1993). The superficial epithelium of the genital ridges is a part of the coelomic epithelium. Proliferation of epithelium leads to the expansion of genital ridge and culminates in the formation of undifferentiated gonad. The undifferentiated gonads are composed of cortex and medulla (Witschi 1929). The cortex contains germ cells enclosed by somatic cells, whereas the medulla contains only somatic cells. The medullar cells assembly into the knots, which are placed metamerically along the gonads. The gonadal metameres are called the gonomeres (Witschi 1929). The anterior gonomeres are bigger and appear earlier than the posterior ones. This indicates that the gonadal development proceeds along antero-posterior axis (Witschi 1929; El Jamil et al., 2008). The developing gonads in Anura are composed of three parts: i) the anterior part (progonad), which differentiates into the fat body; ii) the middle part which forms the proper gonad and iii) the most posterior part (epigonad), which degenerates (Ogielska 2009). The proper gonad develops either into ovary or testes. In the developing ovaries, the germ cells remain in the cortex at the peripheral position and an ovarian cavity forms within the medulla

Abbreviations used in this paper: PGC, primodial germ cell.

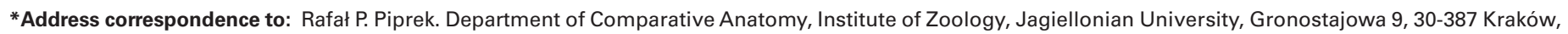
Poland. Phone: +48-1-2664-5059. E-mail: rafal.piprek@uj.edu.pl
}

Supplementary Material (tables) for this paper is available at: http://dx.doi.org/10.1387/ijdb.140155rp

Accepted: 22 January 2015.

ISSN: Online 1696-3547, Print 0214-6282 
(Witschi 1929). The growth of the ovarian cavity and the appearance of oocytes lead to the enhancement of the metamery. In the testes the separation between the cortex and medulla vanishes and the germ cells disperse causing the loss of the metameric structure. The Bufonidae family has a unique morphology of the gonad due to the presence of the Bidder's organs. These organs have an ovary-like structure and develop from the anterior parts of the gonads in males and females (Ogielska and Bartmańska 2009).

In some vertebrates such as caecilians and urodelans the gonads are highly metameric. However, in vertebrates such as anurans and amniotes, the gonads are non metameric, which may be related to the shortening of the whole body length (Handigan and Wassersug 2007). There is little information on the gonadal metamery in anurans but available data show that the number of gonadal metameres differs among species. There are 10-12 gonomeres in Pelobates fuscus and Bufotes viridis and Bombina bombina (Ogielska and Kotusz 2004) and 14 (Chang and Witschi 1956) or 20-30 gonomeres in Xenopus laevis. The discrepancy in the number of gonomers described for $X$. laevis may indicate the variability between individuals and/or between the left and right gonad. In addition, there is significant diversity in the gonadal structure among anurans (Piprek et al., 2010). The goal of the present study was to define whether in anurans, the gonadal metamery is related to the phylogeny or to the gonadal structure. To obtain a broader perspective on the gonadal morphology we studied eight anuran species representing six different families: Bombina bombina (Bombinatoridae), Xenopus laevis (Pipidae) and Pelobates fuscus (Pelobatidae), which represent more basal lineages (Archaeobatrachia), and Hyla arborea (Hyloidae), Bufotes viridis (Bufonoidea) and Rana dalmatina, Rana arvalis, Rana temporarias (Ranoidea), which represent more derived lineages (Neobatrachia) (Fig. 1; Roelants et al., 2007).

\section{Results}

Scanning electron microscopy and light microscopy analyses showed that in all examined anuran species the gonads develop ventrally of the mesonephroi along the dorsal mesentery. However, there were the inter-species differences in the position of the gonad in relation to the mesonephros, the number of gonomeres and the degree of gonadal metamery.

\section{Bombina bombina}

In Bombina bombina the genital ridges developed along almost the entire length of the mesonephroi (Fig. 2A; Supp Table S1). The genital ridges were located in the groove between the two mesonephroi. The undifferentiated gonads were present in Gosner stage 26-33 (G26-33) tadpoles. At these stages, the gonadal metamery was almost undetectable and the anterior parts of the gonads had visible anlagen of the fat bodies.

The differentiating ovaries in B. bombina post G34 tadpoles showed visible external metamery (Fig. 2B). Each ovary was composed of 6 ball-shaped gonomeres containing ovarian cavities (Fig. 2B, insert; Supp Table S1). The gonomers were separated by thin strands of somatic cells (internodal regions). As development progressed the ovaries increased in length and folded (Fig. 2C).

The developing testes after G34 stage gradually lost the metamery, assumed spindle-like shape and became significantly shorter in relation to mesonephroi (Fig. 2D; Supp Table S1). Around metamorphosis (G44-46), the posterior part of testes disappeared, and testes became ovoid (Fig. 2E). From G34 stage onwards, there are two major patterns of gonad length: i. the gonads shorter than a half of mesonephros, and ii. the gonads of almost whole mesonephros length (Supp Table S1).

\section{Xenopus laevis}

In Xenopus laevis the genital ridges (Fig. 2F) formed along almost the entire length of the mesonephroi, however, they were slightly shorter in relation to the mesonephroi than in B. bombina (Supp Table S2). In the undifferentiated gonads (NieuwkoopFaber stages 49-53 which correspond to G27-32) the rudimentary metameres were visible and the gonads were slightly flattened bilaterally (Fig. 2G). The fat bodies were present at the anterior ends of each gonad.

During the ovarian development (after NF53, corresponding to G33), the metameres and the deep intermodal regions (narrowings) dividing the ovaries become clearly visible (Fig. $2 \mathrm{H}$ ). Each ovary contained 14 gonomeres (Supp Table S2). The metamery was clearly visible, and the anterior gonomeres were significantly larger than the posterior ones. At the metamorphosis (NF66, corresponding to G46) the ovaries enlarged and folded (Fig. 2I).

Developing testes lacked external metamery (Fig. 2J). Testes were tape-shaped and bilaterally flattened. Gradually, the posterior ends of tested shortened and disappeared at metamorphosis; testes visibly shortened became ovoid and they were located at the anterior parts of the mesonephroi (Fig. 2K, Supp Table S2). From NF55 (G37) stage onwards, there are two major patterns of gonadal length: visibly short gonads (testes) and long gonads (ovaries) (Supp Table S2). Thus, the gonadal sex is reflected in the gonadal length.

\section{Pelobates fuscus}

In Pelobates fuscus the gonads developed along the anterior half of the mesonephroi (Fig. 2 L,M). The gonads were bead-

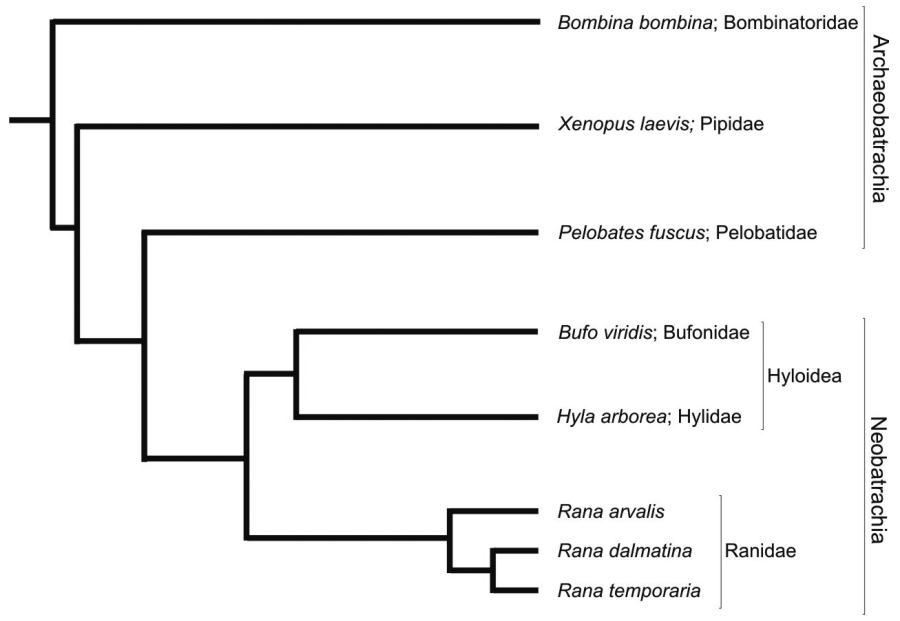

Fig. 1. Phylogeny of studied anuran species. The anuran group is an old and diversified taxon composed of many branches evolved before Jurassic period. The basal groups of anurans such as Archaeobatrachia and more derived groups such as Neobatrachia are separated by long evolutionary period of time. 
shaped and visibly metameric. The gonomeres were joined by narrow internodal regions. Subsequently, the gonomeres grew and became spherical in shape (Fig. 2 L,N). Each gonad had 11 gonomeres and each gonomer contained ovarian cavity (Fig. 2O). The internodal regions lacked ovarian cavity or medullary cells. The cortex of gonomeres and internodal regions contained oogonia and oocytes. However, because the number of germ cells in the gonomeres was higher than in the internodal regions, the cortex of gonomeres was much thicker. The anterior end of each gonad was connected to the fat body (Fig. $2 \mathrm{~L}$ ). The developing testes shortened and lacked external metamery (Supp Table S3). The gonadal sex is easily recognizable: developing testes are much shorter than ovaries.

\section{Hyla aborea}

In Hyla aborea the gonads developed along the anterior half of the mesonephroi (Fig. 3A). Throughout the development only the left gonad developed the fat body in all studied specimens. Along with the growth of the fat body and gonads, the left gonad arched (Fig. $3 \mathrm{~B}, \mathrm{C})$. Thus, in this species the urogenital complex (mesonephros and gonad) was visibly asymmetrical. From the onset of development the tadpole gonads were cylindrical in shape and lacked visible metamery, and the slight clefts present at gonadal surfaces were the only reminder of preexisting gonadal metamery. Due to the lack of external metamery and differences in their length, the sex of the tadpole gonads was morphologically indistinguishable (Supp Table S4).

\section{Bufotes viridis}

In Bufotes viridis the gonads developed along 2/3 part of the mesonephroi (Fig. 3E). Starting from the early tadpoles stage (Gosner stage 33) the gonads were club-shaped owing to development of the Bidder's organs from the anterior part of the gonad (Fig. 3E). Gradually, the gonads and Bidder's organs increased in size, but the external metamery was almost indiscernible (Fig. 3F, Supp Table S5). The external metamery was not pronounced in the tadpole gonads. Only the small folds at the gonadal surface were the signs of external metamery and their presence was related to the formation of medullary knots formed along the gonad axis (Fig. $3 \mathrm{G}, \mathrm{H}$ ). During tadpole development and metamorphosis the gonadal sex was morphologically indistinguishable due to the lack of pronounced external metamery and gonadal length difference.

Rana dalmatina, Rana arvalis, Rana temporaria

In Rana dalmatina, Rana arvalis and Rana temporaria the gonads started developing within about $1 / 2$ of mesonephros length, were located at the anterior end of the mesonephros and extended beyond the mesonephros (Supp Tables S6-S8). In $R$. dalmatina, the anterior $1 / 3$ of the gonad and in $R$. arvalis and $R$. temporaria the half of the gonad was located ahead of mesonephroi (Fig. 4 A-C). In all three Rana species external gonadal metamery was absent and gonadal sex was morphologically indistinguishable during the whole larval development. Histological analysis showed that Rana gonads had vestigial metamery manifested in metamery of ovarian cavities that was present within each medullary knot (Fig. 4 D-F). Thus the gonadal sex can be assessed by gonad internal structure. Within the medullary knots the cortex surrounding ovarian cavity was thin and contained low number of germ cells. The cortex between the knots was thick and abundant in the oocytes (Fig. 4 G,H).
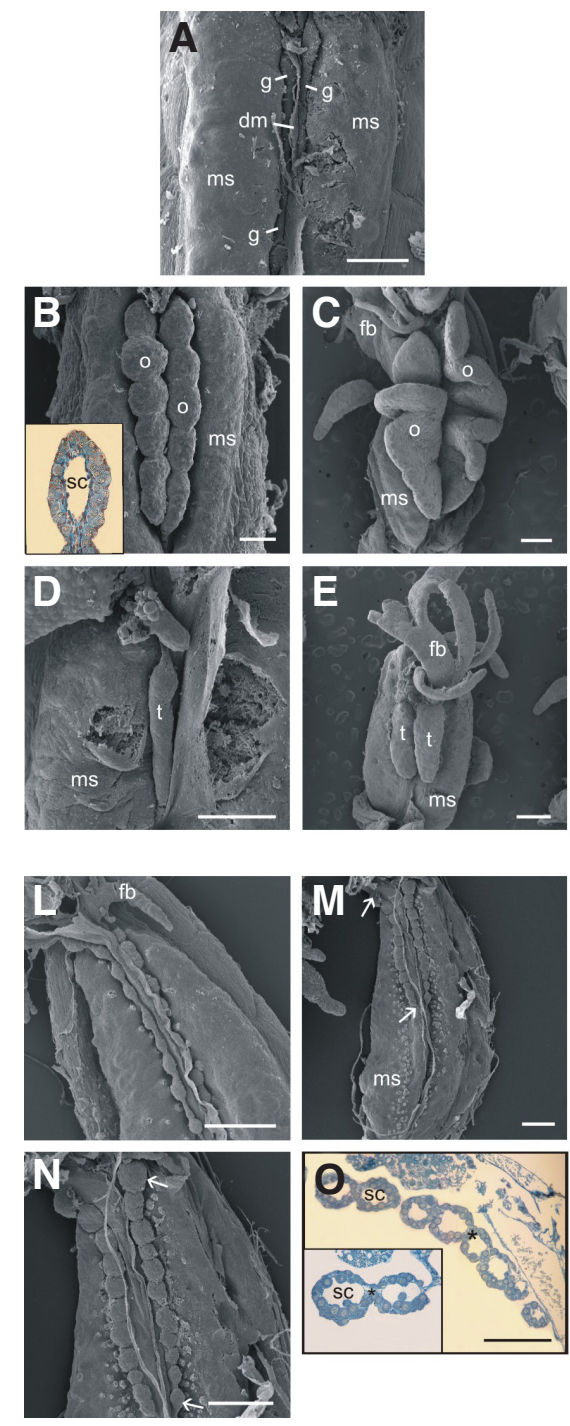
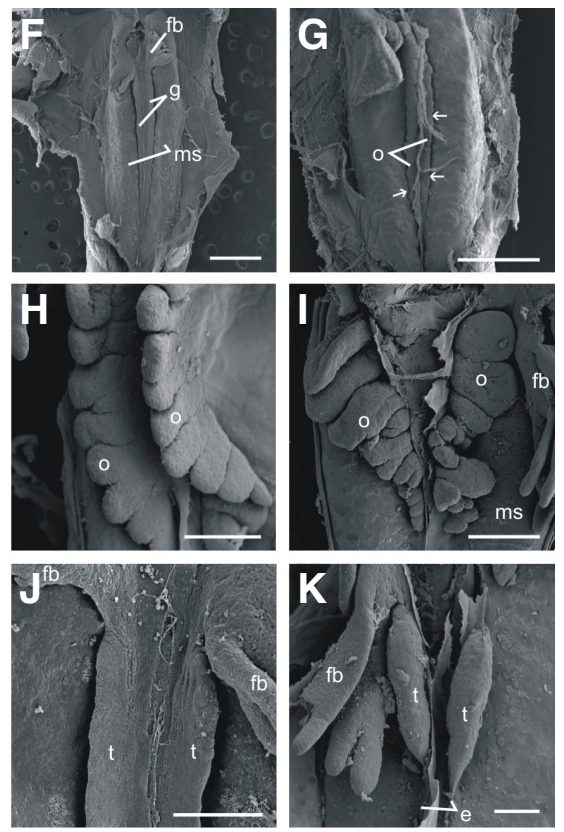

Fig. 2. The morphology of the gonads in (A-E) Bombina bombina, (F-K) Xenopus laevis and (L-O) Pelobates fuscus tadpoles. (A) The undifferentiated gonads (g) of B. bombina tadpole at Gosner stage 30 (G30) are located at the ventral surface of mesonephroi ( $\mathrm{ms}$ ) and are separated from each other by dorsal mesentery (dm). (B) The ovaries (o) of B. bombina tadpole at G37; the insert shows the cross section of the ovarian gonomere containing secondary cavity (sc). (C) The ovaries (o) of B. bombina at the end of metamorphosis (G46); the fat bodies (fb) are situated at the anterior pole of the gonads. (D) The testes ( $t$ ) of B. bombina tadpole at G37. (E) The testes (t) of B. bombina tadpole at the end of metamorphosis. (F) The undifferentiated gonads (g) of X. laevis tadpole at Nieuwkoop-Faber stage 50 (NF50). (G) X. laevis tadpole at stage 58 (NF58). The appearance of the narrowings (arrows) along the gonads indicates their ovarian (o) differentiation. (H) X. laevis tadpole at stage 61 (NF61) with clearly visible ovarian metamery. (I) The ovaries of X. laevis at the end of metamorphosis. (J) The differentiating testes ( $t$ ) of X. laevis tadpole at NF58 lack external metamery. (K) The testes of X. laevis at the end of metamorphosis (NF66); e-epigonad. (L) The ovaries of P. fuscus at G30 stage with clearly visible metameres. (M,N) The ovaries of P. fuscus at G34 stage consist "the string of beads" and occupy half of the mesonephros (ms) length. Arrows point to the anterior and posterior ends of the gonads. (0) The longitudinal section through ovaries of $\mathrm{P}$. fuscus at G44 stage - asterisks indicate the narrowings between gonomeres that contain secondary cavities (sc). Scale bar is equal to $200 \mu \mathrm{m}$. 

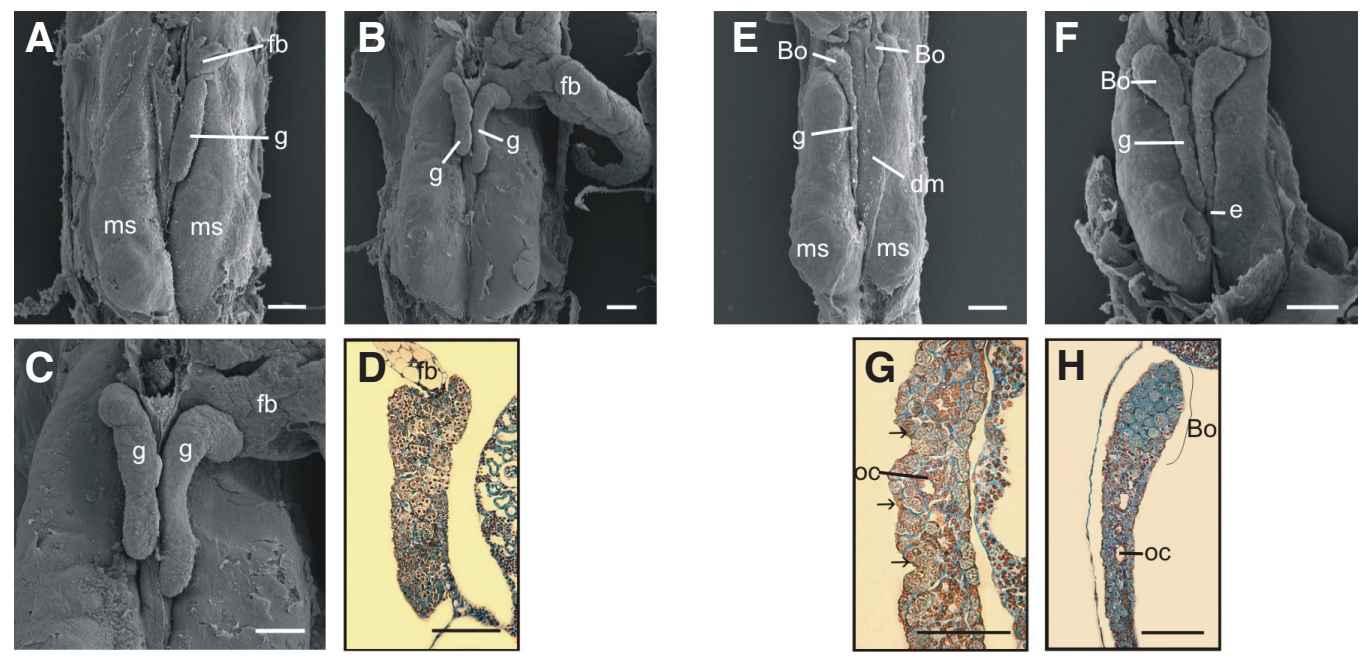

Fig. 3. Gonad morphology in (A-D) Hyla arborea and (E-H) Bufo viridis tadpoles. (A) The gonads of $\mathrm{H}$. arborea at G33 stage; the fat body is presentat the left side; $m s$-mesonephros. (B,C) The gonads of $\mathrm{H}$. arborea at G34 stage; $g$ - gonad. (D) Longitudinal section through the left ovary of $\mathrm{H}$. arborea at G33 stage - no metamery is visible. (E) The Bidder's organs and gonads (g) of B. viridis tadpole at G34 stage - the anterior parts of gonads are enlarged and constitute left and right Bidder's organs (Bo). (F) The gonads of B. viridis tadpole at G37 stage. (G) Longitudinal section of the ovary containing ovarian cavities (oc) and signs of metamery in B. viridis tadpole at G44 stage; the narrowings are indicated by arrows. (H) Longitudinal section of the ovary and Bidder's organ (Bo) in B. viridis tadpole at G44 stage. Scale bar is equal to 200 um.

\section{Discussion}

Our study of several anuran species representing a series (from basal to advanced) of families showed clear inter- and intra-group variability of the gonad morphology but also very well defined evolutionary trends.

The first evolutionary trend is the gradual disappearance of metamery of the gonad. The developing gonads of primitive anurans (Archaeobatrachia such as B. bombina, $X$. laevis and $P$. fuscus) are highly metameric while in more advanced Anura (Neobatrachia) the external metamery is hardly recognizable or absent. However, even in the absence of external metamery, the internal metamery of the gonad (metameric distribution of medul-
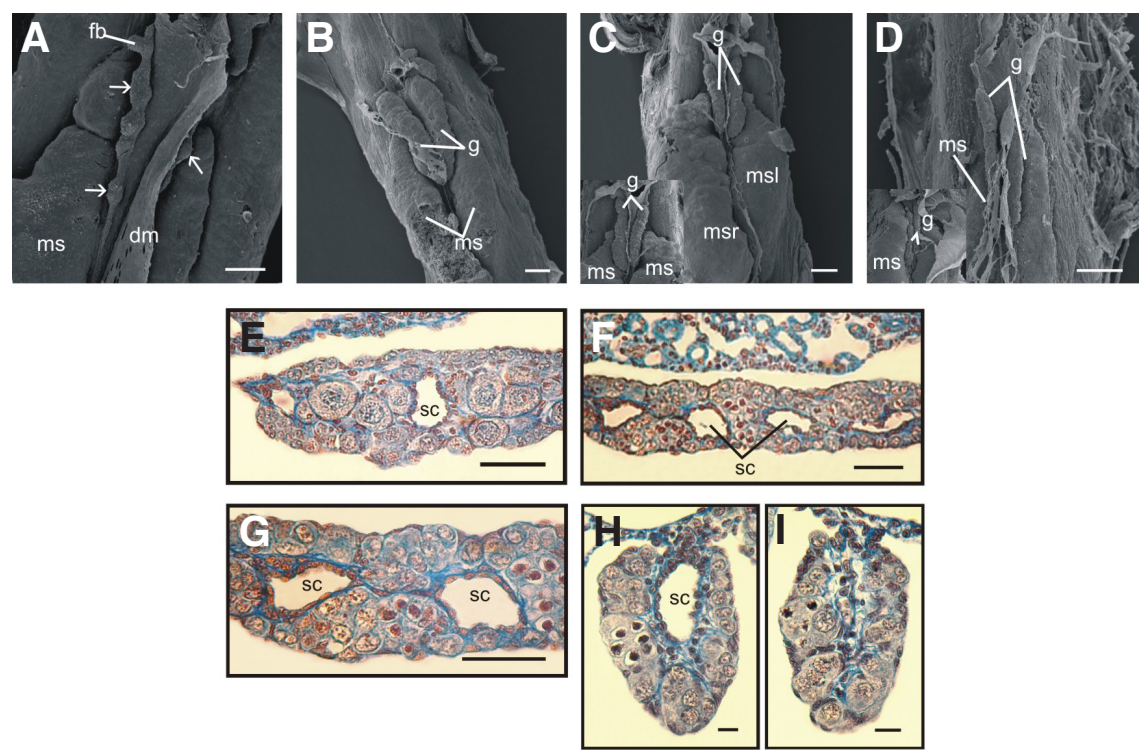

Fig. 4. Gonad morphology in (A) Rana dalmatina at G34 stage, (B) Rana arvalis at G34 stage, and (C) Rana temporaria at G34 stage tadpoles. (D) longitudinal section through R. arvalis ovary at $G 46$ stage, (E,F) longitudinal section through $R$. temporaria ovary at G46 stage, (G) cross section through $\mathrm{R}$. arvalis ovary at the site of gonomere, $\mathbf{( H )}$ cross section through $\mathrm{R}$. arvalis ovary at the site of narrowing. Scale bar is equal to $200 \mu \mathrm{m}$ in A-G and to $30 \mu \mathrm{m}$ in $\mathrm{H}-\mathrm{I}$. lar knots and ovarian cavities) persists in developing gonad. In all studied anuran species the external and internal metamery is lost in differentiating testes. In contrast to Neobatrachia, where the sex of the tadpole gonad cannot be distinguished morphologically, in more primitive Archaeobatrachians there is a striking difference in size and metamery between male and female gonads (elongated metamerized ovaries and short ovoid testes).

The presence of metamery observed in many mesodermal structures seems to be a primitive trait, which gradually disappears during evolution (Goodrich 1913; Opitz 2014). Thus, the presence of gonadal metamery among more primitive anuran lineages and its reduction in more derived groups is consistent with the general evolutionary trend of metamery loss. It is very probable that the lost of gonadal metamery, which occurred during anurans evolution, is related to the progressive shortening of the body length. The caecilians and urodelans have elongated body and highly metamerized gonads, and in anurans there is a gradual shortening of the body and the gonads (Blüm 1986; Exbrayat 2009a,b). The comparative analysis of anuran species may provide valuable insight into the evolution of the metamery in other groups of animals. The genes involved in determination of metamery, especially Hoxgenes, have been well studied in Drosophila (Bilder and Scott 1998). It would be interesting to identify genes involved in the metamery reduction in anurans.

The second evolutionary trend that we noticed was the shortening of the gonads in relation to mesonephroi and change in their location in relation to mesonephroi. In more primitive Archaeobatrachia such as $B$. bombina and $X$. laevis, the gonads developed along almost the entire length of mesonephros. The $P$. fuscus, $H$. arborea and $B$. viridis represent a transitional state where the gonads shorten and occupy only anterior half of the mesonephroi. In Rana, the anterior parts of the gonads are located ahead of the anterior ends 

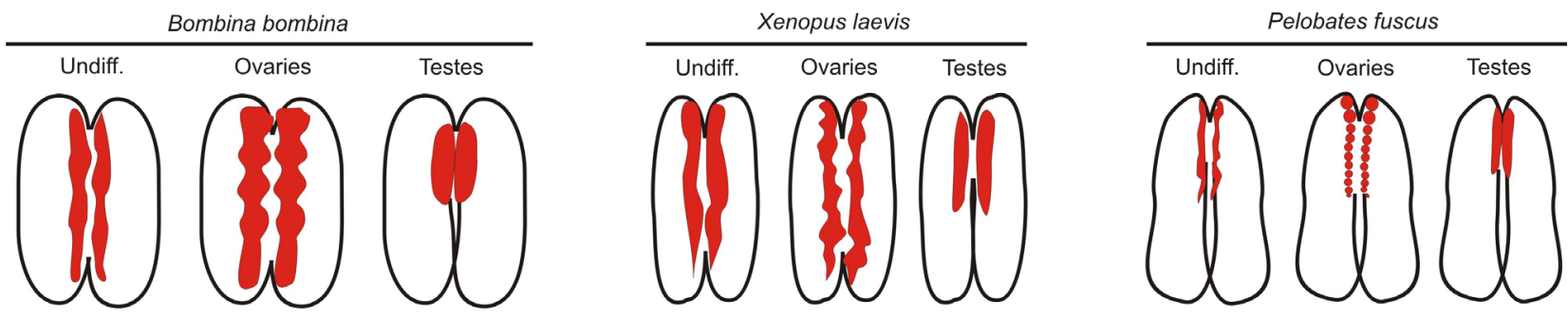

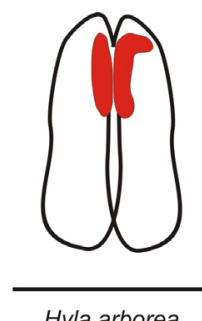

Hyla arborea
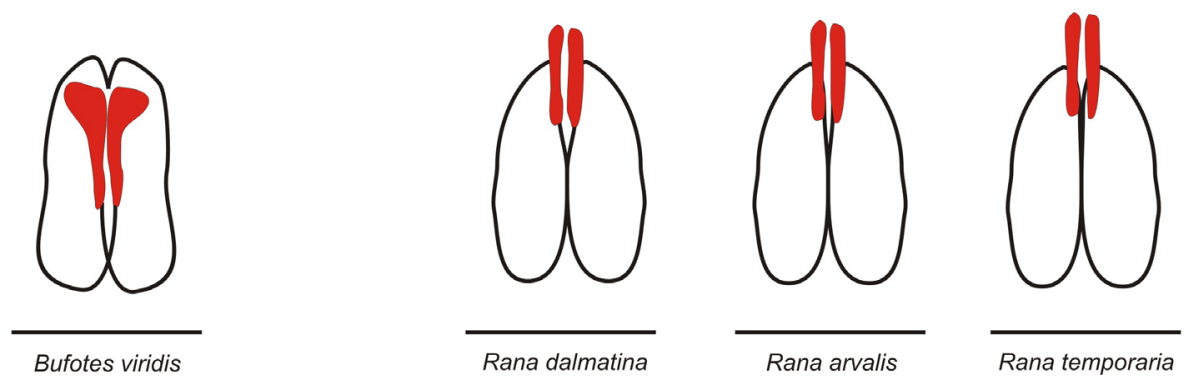

Fig. 5. Scheme of the gonadal morphology in anuran tadpoles. In Bombina bombina, Xenopus laevis and Pelobates fuscus the external structure of the developing gonads allows to distinguish the undifferentiated gonads (Undiff.), ovaries and testes. However, in Neobatrachia the external structure was not sexually differentiated and thus sex of the individuals was not distinguishable by the external morphology. Gonads (red) are presented on the background of mesonephroi.

of the mesonephroi. Such changes in the position of the gonads result from the shift in the location of genital ridge and thus may be related to the shift in the PGCs terminal location site. It is known that in Xenopus, zebrafish, chicken and mouse, the somatic cells, which are present at the PGC terminal location site produce a chemoatractant - the stromal cell-derived factor 1 (SDF1) while the PGCs produce SDF1 receptor CXCR4 (Takeuchi et al., 2010). Thus, the varying placement of SDF1 production sites may define the termination of the PGC migration and determine the site of genital ridge formation. It would be interesting to investigate the sites of chemoatractant synthesis in anuran species and to determine how they correlate with the genital ridge location.

The site of gonad development in relation to the mesonephroi may be important for the physiology of urine production and sperm transport. The sperm exit from the testes through the mesonephroi via kidney tubules and then is released into the Wolffian duct (mesonephric duct). It remains unknown how the sperm transport may influence the urine production and how the urine influences sperm motility and its fertilization potential. Nevertheless, in vertebrates, there is a clear tendency towards the separation of the urinary and reproductive tracts. The first step in such evolutionary changes is a restriction of the sperm tract to a part of the kidney, which is followed by separation of sperm-ducting part of kidney and finally the formation of a separate sperm tract totally bypassing the urinary tract. All these evolutionary trends are clearly visible among anuran amphibians. We observed that in $P$. fuscus, $H$. arborea, $B$. virids, and Rana sp., progressively more anterior part of the mesonephroi became engaged in sperm production and transport. The posterior part of the mesonephroi became enlarged. In the future it would be interesting to study the urine production in the anterior and posterior parts of the mesonephros.

Haczkiewicz and Ogielska (2013) described the process of testes shortening during development in Pelophylax. They showed that the pars gonalis (the middle part) of the genital ridge was composed of 6-9 metameres. During testis development the distal part gradually became reduced and disappeared. The final testis developed from 2-4 anterior gonomeres (Haczkiewicz and Ogielska 2013). The authors concluded that the testes shorten during development. The ovaries, however, develop from the entire length of the gonadal anlagen (Haczkiewicz and Ogielska 2013). This difference is probably related to the difference in size of male and female germ cells, which in turn determine the final volume and surface of the gonad. However, it remains unknown why there is shortening of the genital ridges in both the males and the females during anuran evolution.

We noticed that the urogenital system (mesonephroi and gonads) were more symmetrical in Archaeobatrachia than in Neobatrachia. In Neobatrachia the left gonad was slightly larger than the right one, and the left mesonephros was smaller than the right one. This asymmetry may be related to the presence of a liver at the right site.

In summary our study revealed a great diversity in the gonadal morphology and allowed us to define evolutionary tendencies in the gonadal development. We showed that the metamery of gonads is a primitive feature characteristic for Archaeobatrachia. The gonadal metamery disappeared in evolution, and the gonads shortened and became limited to the anterior parts of the mesonephroi. It would be interesting to investigate whether the gonad shortening is related to the separation of the urinary and reproductive tracts. Future comparative studies are needed to define molecular mechanisms responsible for determination of genital ridge formation sites, and to answer what is the physiology of the diversified kidneys and if/ how the urine production influences the sperm.

\section{Materials and Methods}

Egg clutches of Hyla arborea, Bufotes viridis, Rana arvalis and Rana temporaria were collected in the wild in the vicinity of Bielsko-Biała $\left(49^{\circ} 54^{\prime} \mathrm{N}\right.$ 1903'E; Pogórze Śląskie, Poland); eggs of Bombina bombina and Pelo- 
bates fuscus were collected near Miechów (50²6’ N 2009'E; Wyżyna Miechowska, Poland) and eggs of Rana dalmatina near Tarnów (4958'N $20^{\circ} 49^{\prime} \mathrm{E}$; Pogórze Karpackie, Poland). Tadpoles of Xenopus laevis were raised in the laboratory in 10-L aquaria and fed with boiled dandelion leaves ad libitum. Tadpoles of $X$. laevis were fed with Seramicron (Sera) twice a day. Tadpoles of $X$. laevis were staged according to Nieuwkoop and Faber (1956) and the other species according to Gosner (1960). Tadpoles were anesthetized with MS-222 solution at the sampling points (Supp Tables S1-S8). All specimens were acquired according to Polish legal regulations for the protection of wild species (Dz. U. nr 33, poz. 289, 2005) and with the permission from the Polish Ministry of Environment Protection and Forestry, and approval from the I Local Commission for Ethics in Experiments on Animals.

\section{Light microscopy}

The gonads isolated along with the mesonephroi were fixed in Bouin's solution, dehydrated, embedded in paraplast (Sigma) and sectioned at 6 $\mu \mathrm{m}$. Sections were stained with Debreuill trichrome (Kiernan 1990). Images were taken with a Nikon Eclipse E600 light microscope and processed with Corel Photo-Paint 11.

\section{Scanning electron microscope}

The gonads isolated along with the mesonephroi were fixed in Karnovsky's fixative (Ito and Karnovsky 1968), dehydrated, dried in a LADD critical point drier, fractured and sputter-coated with gold. Samples were viewed with a JEOL JSM 5410 scanning electron microscope at the JagielIonian University Department of Cell Biology and Imagining.

\section{Acknowledgments}

We are grateful to Laboratory of Scanning Electron Microscopy for Biological and Geological Sciences, Institute of Zoology, Jagiellonian University for sharing JEOL JSM 5410 microscope. RPP was supported by a grant from the MNiSzW (N N303 542938) and START stipend from FNP. JZK was supported by a grant from ARC. This publication was supported by funding from the Jagiellonian University within the SET project. The project was co-financed by the European Union.

\section{References}

BILDER D, SCOTT MP (1998). Hedgehog and wingless induce metameric pattern in the Drosophila visceral mesoderm. Dev Biol 201: 43-56.

BLÜM V (Ed.) (1986). Reproduction of Vertebrates. 1st ed, Springer-Verlag, Berlin, New York.

CHANG CY, WITSCHI E (1956). Genetic control and hormonal reversal of sex differentiation in Xenopus. Proc Soc Exp Biol Med 89: 150-152.

EI JAMIL A, MAGRE S, MAZABRAUD A, PENRAD-MOBAYED M (2008). Early aspects of gonadal sex differentiation in Xenopus tropicalis with reference to an antero-posterior gradient. J Exp Zool A 309: 127-137.
EXBRAYAT JM (2009a). Spermatogenesis and male reproductive system in Amphibia - Gymnophiona. In Reproduction of Amphibians (Ed M Ogielska). Science Publishers, Enfield, pp. 125-152.

EXBRAYAT JM (2009b). Oogenesis and female reproductive system in Amphibia - Gymnophiona. In Reproduction of Amphibians (Ed M Ogielska). Science Publishers, Enfield, pp. 305-342.

GOODRICH ES (1913). Metameric segmentation and homology. Quart J Micr Sci 59: $227-248$.

GOSNER LK (1960). A simplified table for staging anuran embryos and larvae with notes on identification. Herpetologica 16: 513-543.

HACZKIEWICZ K, OGIELSKA M (2013). Gonadal sex differentiation in frogs: how testes become shorter than ovaries. Zool Sci 30: 125-134.

HANDIGAN GR, WASSERSUG RJ (2007). The anuran Bauplan: a review of the adaptive, developmental, and genetic underpinnings of frog and tadpole morphology. Biol Rev Camb Philos Soc 82: 1-25.

ITO S, KARNOWSKY MJ (1968). Formaldehyde glutaraldehyde fixatives containing trinitro compounds. J Cell Biol 36: 168.

KIERNAN JA (Ed.) (1990). Histological and Histochemical Methods: Theory and Practice. 2nd ed, Pergamon Press, Oxford, New York, Seoul, Tokyo.

NIEUWKOOP PD, FABER J (Eds.) (1956). Normal tables of Xenopus laevis (Daudin). 1st ed Amsterdam, North-Holland.

OGIELSKAM (2009). Undifferentiated amphibian gonad. In Reproduction of Amphibians (Ed M Ogielska). Science Publishers, Enfield, pp 1-33.

OGIELSKA M, BARTMAŃSKA J (2009). Spermatogenesis and male reproductive system in Amphibia-Anura. In Reproduction of Amphibians (Ed M Ogielska). Science Publishers, Enfield, pp 34-99.

OGIELSKA M, KOTUSZ A (2004). Pattern and rate of ovary differentiation with reference to somatic development in anuran amphibians. J Morph 259: 41-54.

OPITZ JM (2014). Counselling in Cases of Idiopathic Syndromes. In: Clinical Genetics: Problems in Diagnosis and Counselling. (Eds AM Willey, TP Carter, S Kelly). New York, Academic Press, pp. 153-169.

PIPREK RP, PECIO A, SZYMURA JM (2010). Differentiation and development of gonads in the yellow-bellied toad, Bombina variegata L. 1758 (Amphibia: Anura: Bombinatoridae). Zool Sci 27: 47-55.

ROELANTS K, GOWER DJ, WILKINSON M, LOADER SP, BIJU SD, GUILLAUME K MORIAU L, BOSSUYT F (2007). Global patterns of diversification in the history of modern amphibians. Proc Natl Acad Sci USA 104: 887-892.

TAKEUCHI T, TANIGAWA Y, MINAMIDE R, IKENISHI K, KOMIYA T (2010). Analysis of SDF-1/CXCR4 signaling in primordial germ cell migration and survival or differentiation in Xenopus laevis. Mech Dev 127: 146-158.

WITSCHI E (1929). Studies on sex differentiation and determination in amphibians I. Development and sexual differentiation of the gonads of Rana sylvatica. J Exp Zool 52: 235-265.

WYLIE CC, BANCROFT M, HEASMAN J (1976). The formation of the gonadal ridge in Xenopus laevis. II. A scanning electron microscope study. J Embryol Exp Morph 35: 139-148.

WYLIE CC, HEASMAN J (1993). Migration, proliferation, and potency of primordial germ cells. Semin Dev Biol 4: 161-170. 


\section{Further Related Reading, published previously in the Int. J. Dev. Biol.}

On the transition from the meiotic to mitotic cell cycle during early mouse development Jacek Z. Kubiak, Maria A. Ciemerych, Anna Hupalowska, Marta Sikora-Polaczek and Zbigniew Polanski Int. J. Dev. Biol. (2008) 52: 201-217

http://dx.doi.org/10.1387/ijdb.072337jk

P450 aromatase expression in the temperature-sensitive sexual differentiation of salamander (Hynobius retardatus) gonads Natsuko Sakata, Yoichiro Tamori And Masami Wakahara

Int. J. Dev. Biol. (2005) 49: 417-425

http://dx.doi.org/10.1387/ijdb.041916ns

Germ line development in fishes

A K Braat, J E Speksnijder and D Zivkovic

Int. J. Dev. Biol. (1999) 43: 745-760

http://dx.doi.org/10.1387/ijdb.10668983

Primordial germ cell development: is the urodele pattern closer to mammals than to anurans?

M Wakahara

Int. J. Dev. Biol. (1996) 40: 653-659

http://dx.doi.org/10.1387/ijdb.8877437

Determination of the sensitive stages for gonadal sex-reversal in Xenopus laevistadpoles I Villalpando and $\mathrm{H}$ Merchant-Larios

Int. J. Dev. Biol. (1990) 34: 281-285

http://dx.doi.org/10.1387/ijdb.2386730

5 yr ISI Impact Factor $(2013)=2.879$
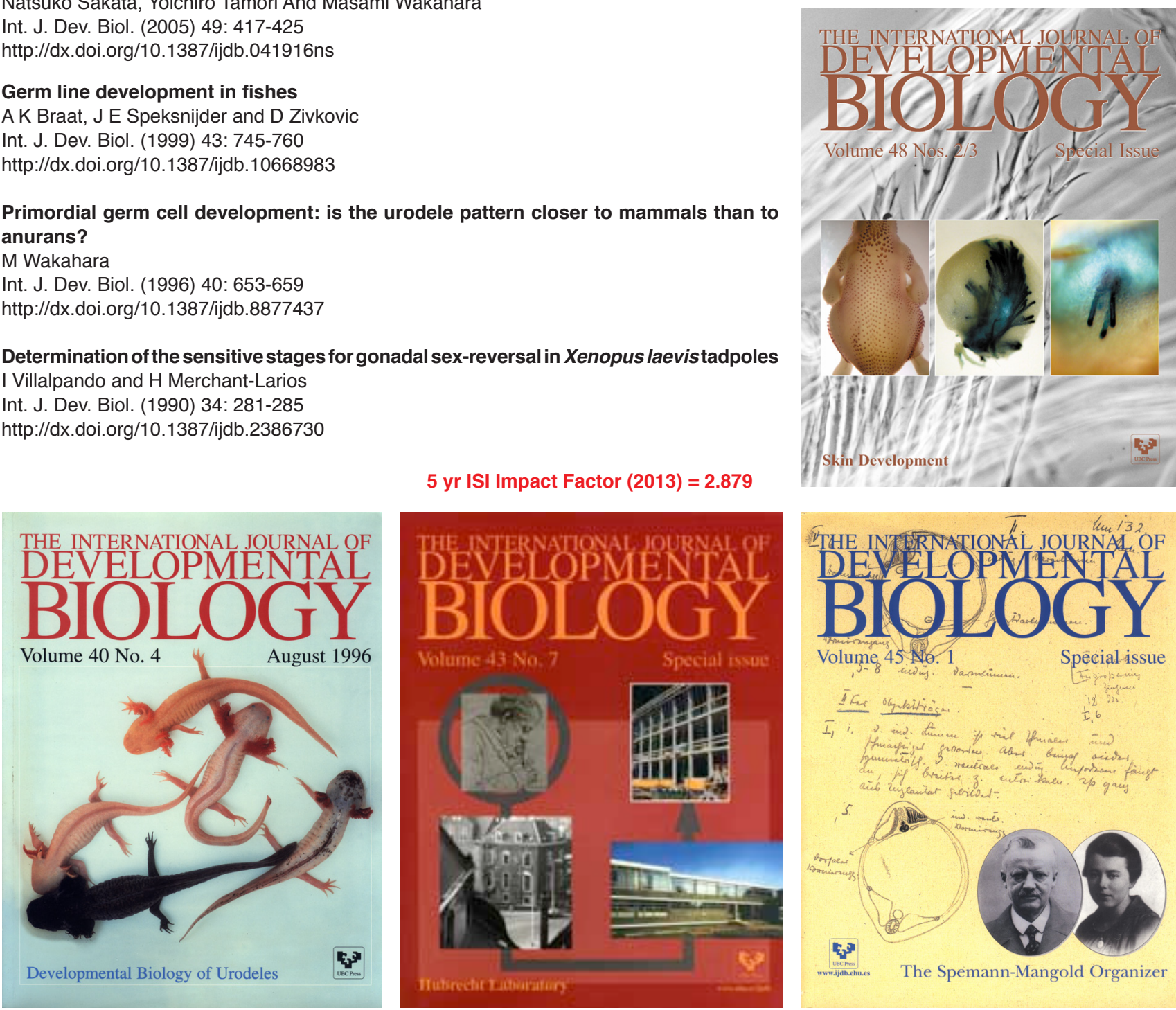

Volume 45 No. 1

Special issue

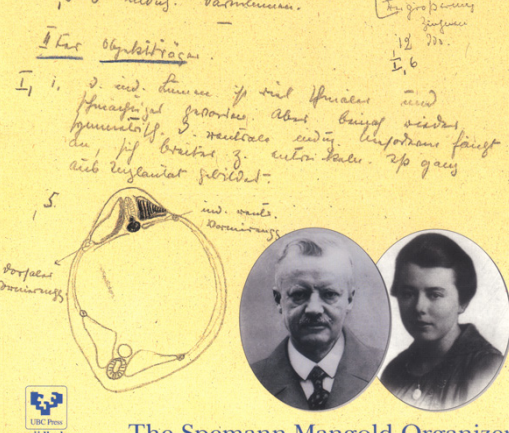

6ु?

The Spemann-Mangold Organizer 